\title{
AOR
}

Selected Papers of \#AolR2020:

The $21^{\text {st }}$ Annual Conference of the

Association of Internet Researchers

Virtual Event / 27-31 October 2020

\section{VACCINE INFORMATION SEEKING AND SHARING: HOW PRIVATE FACEBOOK GROUPS CONTRIBUTED TO THE ANTI-VACCINE MOVEMENT ONLINE}

\author{
Kolina Koltai \\ University of Washington

\section{Introduction}

A major factor in vaccine dissent is the proliferation of vaccine opposed content online (Kata, 2012). The affordances of the internet have contributed to the rise of alleged conspiracies (Fahnestock, 2016), like the link between autism and vaccines, which is further amplified through social media and bots (Broniatowski et al., 2018). The widespread availability of misinformation has been an integral part of the vaccine opposed movement's success. They have been highly effective in spreading their messages and arguments through social media and content sharing platforms (Wilson \& Keelan, 2013). People also seek out and join digital communities to gain support and health advice from others similar to them (Zhang, He, \& Sang, 2013).

This leads to the research question guiding the work: How do anti-vaccine Facebook users use social media to search and evaluate vaccine related information and communities? With the increase of members joining anti-vaccine Facebook groups online, it is critical to understand how these users are utilizing the platform to find and share vaccine opposed information, especially during shifting policy changes by Facebook. In 2019, Facebook implemented a series of algorithmic changes to limit the spread of vaccine opposed content and groups on their platform; however, membership continues to grow.

\section{Methods and Analysis}

This study was part of a larger body of work conducted by the author. The findings discussed in this paper draw primarily from interviews and data collected from AntiVaccine Facebook Groups (AVFBG). Interview participants, $(n=15)$ were recruited from Suggested Citation (APA): Koltai, K. (2020, October 28-31). Vaccine information seeking and sharing: How private Facebook groups contributed to the Anti-vaccine movement online. Paper presented at AolR 2020: The $21^{\text {st }}$ Annual Conference of the Association of Internet Researchers. Virtual Event: AolR. Retrieved from http://spir.aoir.org. 
an earlier survey distributed in AVFBGs. Interviews, conducted October - December 2019, revolved around their vaccine attitudes, their values, and their use of Facebook as an information seeking, information sharing, and community building tool. Data was also collected from 20 AVFBGs by screenshotting the 30 most recent posts in those groups resulting in approximately 600 posts, collected in December 2019. Thematic analysis (Braun \& Clarke, 2006) was used to analyze both the interviews and vaccine content shared on Facebook.

\section{AVFBGs are information exchange resources hubs}

AVFBGs have become a vital resource in how people find and share vaccine opposed information. In the interviews, participants described not being satisfied with the vaccine information they were receiving from their healthcare provider, and in many cases, having their concerns dismissed. Their health information wants (Xie, 2009) were not being fulfilled so they sought out other information sources. They not only found information that aligned with their values, but also a welcoming environment of likeminded members who shared their concerns. Common information behaviors in AVFBGs are users requesting vaccine information (e.g., asking for sources about the ingredients in vaccines) and sharing vaccine related information (e.g., news article about vaccines). It gives users an easy way to quickly distribute information that would be appealing to the community.

\section{AVFBGs are not a total echo chamber}

A common assumption of the AVFBGs is that members are in an echo chamber. An echo chamber is when a group of participants chooses to preferentially connect with each other while excluding others with outside views (Bruns, 2017). Interestingly, members share pro-vaccine information in AVFBGs. While the intention of sharing provaccine information is to be critical of it, members in these digital spaces regularly encounter pro-vaccine content both in their regular feeds and the AVFBGs. With the sharing of pro-vaccine information, members in these closed digital communities are still being exposed to outside views. The members of these communities consistently interact, even actively engage, with viewpoints different from their own, both online and in-person.

\section{Facebook's insufficient moderation of AVFBGs}

Due to pressure to address misinformation on the platform, Facebook has enacted policies to minimize the presence of vaccine opposed information. Moderation of content on social media platforms is a difficult task as you need to enforce changing social norms of the community (Gillespie, 2018). In this study, participants described being well aware of Facebook's policies and began developing their own ways to adapt to the censorship through their understanding of the algorithm as well as mimicking how other members were adapting. Members created workarounds to censorship like using non-standard lexical variations on the word 'vaccine' (e.g., 'va((ine'), renaming groups to new terminology (e.g., instead of 'anti-vaccine', using 'health freedom'), using screenshots to share information instead of links, and even migrating information and communities to other platforms (e.g., MeWe and private forums). There is evidence to suggest that just like legal policy changes (e.g., removing vaccine exemptions), digital policy changes are not enough to encourage the vaccine opposed to vaccinate. AVFBG 
members will develop workarounds and may become more confident in their decision not to vaccinate.

\section{AVFBGs foster community and belonging}

These digital vaccine opposed communities, despite being demoted in Facebook's search results, keep growing in membership. They function both as an information exchange hub but also as a social support network. Members have joined in search of vaccine information, but they stayed because they found a sense of community among strangers. Participants described that the people in these groups do connect over having similar values and concerns about vaccines. They form these network ties due to homophily through values. These values also appear to be consistent group-to-group. When collecting the Facebook posts, often the same resource or the same article would be posted multiples times across the groups and even in the same group by different members. These communities become incredibly important as members get more involved in fighting against vaccine mandates.

In the interviews, members often described the relief and happiness they experience in these groups because they do not need explain the choice not to vaccinate. There is validation of vaccine opposition and a support system to handle the constant criticism (or hiding) of your vaccine attitudes from the public. To protect these spaces and their children, these communities foster a collectivistic environment that translates into taking direct actions to help the group (e.g., protesting). These spaces continue to be powerful because they encourage vaccine refusal and taking action to protect that refusal for your own family and others.

\section{References}

Braun, V. \& Clarke, V. (2006). Using thematic analysis in psychology. Qualitative research in psychology, 3(2), 77-101.

Bruns, A. (2017). Echo Chamber? What Echo Chamber? Reviewing the Evidence. Paper presented at 6th Biennial Future of Journalism Conference, Cardiff, UK.

Broniatowski et al. (2018). Weaponized health communication: Twitter bots and Russian trolls amplify the vaccine debate. American Journal of Public Health, 108(10), 13781384.

Fahenstock, J. (2017). Controversies on the web: The case of adult human neuogenesis. In A. G. Gross, \& J. Buehl (Eds.), Science and the Internet: Communicating Knowledge in a Digital Age (pp. 115-141). Taylor \& Francis.

Gillespie, T. (2018). Custodians of the Internet: Platforms, content moderation, and the hidden decisions that shape social media. Yale University Press.

Kata, A. (2012). Anti-vaccine activists, Web 2.0, and the postmodern paradigm-An overview of tactics and tropes used online by the anti-vaccination movement. Vaccine, 30(25), 3778-3789. 
Wilson, K., \& Keelan, J. (2013). Social media and the empowering of opponents of medical technologies: The case of anti-vaccinationism. Journal of Medical Internet Research, 15(5), e103.

Xie, B. (2009). Older adults' health information wants in the Internet age: Implications for patient-provider relationships. Journal of Health Communication, 14(6), 510-524.

Zhang, Y., He, D., \& Sang, Y. (2013). Facebook as a platform for health information and communication: A case study of a diabetes group. Journal of Medical Systems, 37(3), 207-12. 